\title{
REGULADORES VEGETAIS NA CONSERVAÇÃO PÓS-COLHEITA DE GOIABAS 'PALUMA'1
}

\author{
MARIA APARECIDA LIMA², JOSÉ FERNANDO DURIGAN ${ }^{3}$
}

\begin{abstract}
RESUMO - Avaliaram-se os efeitos da aplicação exógena de solução de ácido giberélico (GA), 6-benzilaminopurina (6-BAP) ou ácido indol-3-acético (IAA) a 100 ou $200 \mathrm{mg} \mathrm{L}^{-1}$ e de $\mathrm{CaCl}_{2}$ a $1 \%$ ou 2\%, na conservação pós-colheita de goiabas-'Paluma', por infiltração a vácuo $\left(500 \mathrm{mmHg} .20\right.$ minutos $\left.^{-1}\right)$. Os frutos tratados foram armazenados ao ambiente $\left(21,6^{\circ} \mathrm{C}, 73,4 \% \mathrm{UR}\right)$ e analisados, periodicamente, física e quimicamente. Os tratamentos com cloreto de cálcio a $1 \%$ ou $2 \%$ foram os melhores na conservação destas goiabas, pois propiciou-lhes vida útil comercial de 7 dias, o que corresponde a aumento de um dia na vida de prateleira, quando comparados com os demais tratamentos. Os tratamentos utilizados não apresentaram efeitos significativos na evolução da coloração e da firmeza dos frutos durante o amadurecimento.
\end{abstract}

Termos para indexação: auxina, citocinina, giberelina, cálcio, armazenamento.

\section{GROWTH REGULATORS ON 'PALUMA' GUAVAS POSTHARVEST CONSERVATION}

ABSTRACT - The effects of the application effects of gibberelic acid, 6-benzilaminopurine (6-BAP), and indol-3-acetic acid (IAA) at 100 or $200 \mathrm{mg} \mathrm{L}^{-1}$ and calcium chloride at $1 \%$ or $2 \%$ by vacuum infiltration ( $500 \mathrm{mmHg} .20$ minute-1), on postharvest 'Paluma' guavas were studied. The fruits were stored at $21,6^{\circ} \mathrm{C}, 73,4 \% \mathrm{UR}$ and periodically analyzed physic and chemically. The treatments with $1 \%$ or $2 \% \mathrm{CaCl}_{2}$ were the best with 'Paluma' guavas, showing a shelflife of 7 days, increasing its in one day, when compared with other treatments. These treatments did not affect significantly the evolution of color and firmess during the ripening.

Index terms: auxin, citokinin, gibberelic acid, calcium, storage.

\section{INTRODUÇÃO}

A goiaba tem pequena vida útil pós-colheita, ou seja, de apenas 3 dias, quando mantida em ambiente a $25-30^{\circ} \mathrm{C}$ (Durigan, 1997). Os principais fatores depreciadores de sua qualidade na pós-colheita são a rápida perda da coloração verde da casca, o amolecimento, a incidência de podridões , o murchamento e a perda de brilho (Jacomino et al., 2001).

Siddiqui \& Bangerth (1996) citam que o cálcio participa de forma importante na estrutura e na resistência mecânica da parede celular, facilitando ligações entre polímeros de pectina da lamela média, o que aumenta esta resistência (Chitarra \& Chitarra, 1990). Este elemento também controla o processo de desintegração da mitocondria, do retículo endoplasmático e da membrana citoplasmática, reduzindo a taxa respiratória. Sua aplicação exógena pode contribuir para aumentar a vida pós-colheita de muitas frutas, segundo o revisado por Durigan (1997).

Brady (1987) relata que o amolecimento faz parte do amadurecimento de quase todos os frutos e tem uma enorme importância comercial, pois, quando acontece muito rapidamente, limita a vida pós-colheita dos mesmos, facilitando o aumento de injúrias mecânicas durante o manuseio e aumentando a suscetibilidade a doenças. Normalmente, o amolecimento é acompanhado de aumento na concentração de pectina solúvel.
Vasquez-Ochoa \& Colinas-Leon (1990) observaram, em goiabas, que a resistência da textura diminui com o amadurecimento, evoluindo de 25,0 N no fruto "de vez" para 5,0 N no "muito maduro".

Pathmanaban et al. (1995) observaram que frutos de goiaba, tratados por imersão em solução de $\mathrm{CaCl}_{2} \mathrm{a} 4 \%$, por 1 hora, e embalados em sacos plásticos contendo $\mathrm{CaCl}_{2}$ na estrutura, quando armazenados ao ambiente, apresentaram retardo no amadurecimento, no desenvolvimento da coloração e na perda de firmeza.

O etileno e o ácido abscísico são tidos como promotores do amadurecimento em frutos, enquanto as giberelinas, as auxinas, as citocininas e os íons cálcio como inibidores (Chitarra \& Chitarra, 1990).

A aplicação de auxina a frutos, na forma de 2,4D, por imersão ou infiltração a vácuo, permitiu a Vendrell (1985) observar, em tomate e banana, que ocorreu um aumento na produção de etileno, mas que também houve um retardo no amadurecimento, e que este último efeito prevaleceu, dependendo da distribuição e da concentração da auxina.

Teaotia et al. (1972) relatam que o ácido giberélico, quando aplicado em pós-colheita, teve efeito retardador ao amadurecimento de goiabas, reduzindo-lhes a taxa de respiração e as mudanças na coloração.

Este trabalho teve como objetivo avaliar a ação de regu-

1 (Trabalho 135/2001). Recebido: 30/08/2001. Aceito para publicação: 06/06/2002.

2 Aluna de Doutorado mplima @ fcav.unesp.br, bolsista FAPESP, Departamento de Tecnologia FCAV/UNESP, Via de acesso Prof. Paulo Donato Castellane, 14.884-900, Jaboticabal, SP, Brasil.

3 Professor Titular jfduri@fcav.unesp.br, Departamento de Tecnologia FCAV/UNESP, Via de acesso Prof. Paulo Donato Castellane, 14.884-900, Jaboticabal, SP, Brasil. 
ladores do amadurecimento (giberelinas, auxinas e citocininas) e do cálcio, na vida útil de goiabas recém-colhidas, quando aplicados exogenamente.

\section{MATERIAL E MÉTODOS}

Utilizaram-se frutos “de vez”, o que corresponde à coloração verde-mate (Pereira, 1995), colhidos em outubro de 1999, pela manhã, em Vista Alegre do Alto-SP, e imediatamente transportados ao Laboratório de Tecnologia dos Produtos Agrícolas da UNESP - Jaboticabal-SP. Os tratamentos aplicados foram diferentes soluções de manitol a $300 \mathrm{mM}$ contendo os reguladores ácido giberélico, ácido indol-3-acético (IAA), 6-benzilaminopurina (6-BAP) a 100 mg.L $\mathrm{L}^{-1}$ ou $200 \mathrm{mg} \cdot \mathrm{L}^{-1} \mathrm{e} \mathrm{CaCl}_{2}$ a $1 \%$ ou $2 \%$.

Utilizaram-se 64 frutos por tratamento, divididos em 16 lotes, contendo 4 frutos cada. Os frutos foram tratados com as soluções, por 20 minutos, sob condição de vácuo $(500 \mathrm{mmHg})$, conforme o sugerido por Frenkel et al. (1969). A solução para imersão dos frutos estava contida em dessecador, o qual tinha acoplado em sua tampa uma bomba de vácuo.

Após receberem os tratamentos, os frutos foram armazenados ao ambiente $\left(21,6^{\circ} \mathrm{C}, 73,4 \% \mathrm{UR}\right)$ e analisados periodicamente quanto às suas características físicas e químicas. Os 16 lotes que integravam cada tratamento, foram assim distribuídos: 4 para avaliações físicas e denominados, controle, e 12 para serem amostrados a cada 2 dias, com 2 repetições, e analisados física e quimicamente.

Os frutos do controle eram avaliados diariamente, quanto à perda de massa fresca, aparência, presença de podridões e taxa respiratória. A aparência foi avaliada, segundo uma escala de pontos, onde: 1=ótimo e 5=totalmente murcho, tendo-se como limite para uso comercial a nota 3,0 (Lima, 1999). A presença de podridões foi relatada pela porcentagem de frutos doentes em relação ao total de frutos avaliados, através de observação visual e identificação dos patógenos no Laboratório de Fitopatologia da UNESP - Jaboticabal-SP, através de observação das estruturas do agente em microscópio óptico comum e comparação destas com o apresentado por Barnett \& Hunter (1972). A taxa respiratória dos frutos foi medida mantendo-se os frutos em recipiente hermeticamente fechado, durante uma hora, do qual se tomaram alíquotas de $0,3 \mathrm{~mL}$ de ar, antes e depois deste tempo, nas quais se determinou o conteúdo de $\mathrm{CO}_{2}$, utilizando-se de Cromatógrafo Finnigan 9001.

Os frutos amostrados eram avaliados quanto à coloração e firmeza, e depois de triturados, embalados em sacos plásticos e estocados a $-18^{\circ} \mathrm{C}$, para posterior determinação dos teores de ácido ascórbico e pectinas (total e solúvel).

A coloração foi determinada utilizando-se de um reflectômetro Minolta Croma Meter CR-200b, o que permitiu relatála através do ângulo hue ou de cor. A firmeza foi determinada, utilizando-se de penetrômetro Bishopâ FT 327 com ponteira de 8 $\mathrm{mm}$, através de leituras, nas laterais opostas dos frutos. Os conteúdos de ácido ascórbico foram determinados segundo a metodologia da AOAC (1997).

Os teores de pectina total e solúvel foram determinados em extrato obtido conforme a metodologia de McCready \& McComb (1952), segundo técnica adaptada de Blumenkrantz \& Asboe-Hansen (1973).
A evolução da massa fresca foi analisada estatisticamente, através de regressão polinomial (Gomes, 1977), e as equações de $1^{\circ}$ grau comparadas quanto ao paralelismo através do teste $t$, conforme o proposto por Neter et al.(1978), utilizando-se do programa RECOM. A análise dos demais dados foi feita, utilizandose de um delineamento inteiramente casualizado, com 2 repetições, através de um esquema fatorial 10x5 (10 tratamentos e 5 datas de análise).

\section{RESULTADOS E DISCUSSÕES}

A massa fresca dos frutos, durante o período de armazenamento, decresceu de maneira constante e significativa em todos os tratamentos. Os frutos submetidos ao tratamento com $\mathrm{CaCl}_{2}$ a $2 \%$ apresentaram a menor perda, enquanto os submetidos ao 6-BAP a 200mg. $\mathrm{L}^{-1}$ apresentaram as maiores (Tabelas 1 e 2).

A vida útil das goiabas submetidas aos tratamentos $\mathrm{CaCl}_{2}$ a $1 \%$ e a $2 \%$ foi de 7 dias (nota 3), enquanto para os submetidos aos demais tratamentos foi de 6 dias. Neste período, não se observou o aparecimento de doenças, que só surgiram no $8^{\circ}$ dia, nos frutos submetidos ao tratamento IAA $200 \mathrm{mg} . \mathrm{L}^{-1}$, e no $9^{\circ}$ dia, nos do GA 200 mg.L-1 (Tabela 3). Nestas datas, os frutos apresentavam-se senescentes, e o aparecimento de doenças deve estar relacionado com o amolecimento devido ao processo natural de envelhecimento. A análise dos frutos doentes indicou que a doença incidente era a antracnose, causada pelo fungo Colletotrichum sp.

A evolução externa dos frutos (Tabela 4) submetidos a todos os tratamentos evoluiu de verde para amarelo. $\mathrm{O}$ ângulo hue diminuiu, em média, de 117,0 (verde) no $1^{\circ}$ dia para 93,1 (amarelo) no $7^{\circ}$ dia. Não se observaram diferenças significativas entre os tratamentos durante o tempo de armazenamento. $\mathrm{O}$ efeito retardador da giberelina e da citocinina na evolução da coloração, devido a atraso na degradação da clorofila, conforme o relatado por Ludford (1995) e Chitarra \& Chitarra (1990), não foi observado.

A firmeza destes frutos diminuiu, em todos os tratamentos, como resultado do amadurecimento, evoluindo de $97,3 \mathrm{~N}$, no $1^{\circ}$ dia, para $19,6 \mathrm{~N}$, no $7^{\circ}$ dia, sem ser influenciada pelos tratamentos (Tabela 5). Não se observou o efeito retardador do íon cálcio, preconizado por Siddiqui \& Bangerth (1996) e Pathmanaban et al. (1995).

O conteúdo de pectina total nos frutos, que no $1^{\circ}$ dia era de $0,89 \mathrm{~g}$ de ácido urônico. $100 \mathrm{~g}$ de polpa ${ }^{-1}$, reduziu-se para 0,53 $0,75 \mathrm{~g}$, após 7 dias, como resultado do processo de envelhecimento. O conteúdo de pectina solúvel, no entanto, foi crescente ao longo do período de armazenamento em todos os tratamentos (Tabela 6). Pode-se observar que a evolução desta solubilização foi mais lenta nos frutos submetidos aos tratamentos $\mathrm{CaCl}_{2} \quad 1 \%$ e GA $200 \mathrm{mg} . \mathrm{L}^{-1}$ até o $5^{\circ}$ dia de armazenamento, enquanto nos demais tratamentos essa evolução foi crescente e irregular. Não se observou relação entre a firmeza e este conteúdo de pectina solúvel.

O conteúdo de ácido ascórbico, Tabela 7, que era de 78,5 mg.100g de polpa ${ }^{-1}$ no $1^{\circ}$ dia, diminuiu durante o armazenamento, o que também foi observado por Lima \& Durigan (2000). Os frutos submetidos aos tratamentos com ácido indol-3-acético 
TABELA 1 - Equações de regressão obtidas para a evolução da massa fresca, em função do tempo de armazenamento (9 dias) ao ambiente $\left(21,6^{\circ} \mathrm{C} ; 73,4 \% \mathrm{UR}\right)$, de goiabas-'Paluma' submetidas a diferentes tratamentos.

\begin{tabular}{|c|c|c|}
\hline Tratamentos & $\mathrm{Y}=\mathrm{A}-\mathrm{BX}^{\mathrm{b}}$ & $\mathrm{R}$ \\
\hline Testemunha & $Y=101,7881-2,0852 X$ & $-0,9959 * *$ \\
\hline Manitol & $Y=100,8300-2,1360 X$ & $-0,9898 * *$ \\
\hline $\mathrm{CaCl}_{2} 1 \%$ & $Y=101,5846-2,0050 X$ & $-0,9960 * *$ \\
\hline $\mathrm{CaCl}_{2} 2 \%$ & $Y=101,3439-1,7937 X$ & $-0,9968 * *$ \\
\hline GA $100 \mathrm{mg} . \mathrm{L}^{-1}$ & $Y=101,3867-1,9347 X$ & $-0,9974 * *$ \\
\hline GA $200 \mathrm{mg} \cdot \mathrm{L}^{-1}$ & $Y=101,6664-2,2402 X$ & $-0,9964 * *$ \\
\hline 6-B AP $100 \mathrm{mg} \cdot \mathrm{L}^{-1}$ & $Y=101,9850-2,0610 X$ & $-0,9980 * *$ \\
\hline 6-B AP $200 \mathrm{mg} . \mathrm{L}^{-1}$ & $Y=104,4369-3,1478 X$ & $-0,9908 * *$ \\
\hline IAA $100 \mathrm{mg} \cdot \mathrm{L}^{-1}$ & $Y=101,5642-2,1622 X$ & $-0,9935 * *$ \\
\hline IAA $200 \mathrm{mg} . \mathrm{L}^{-1}$ & $Y=101,6072-2,1617 X$ & $-0,9935 * *$ \\
\hline
\end{tabular}

${ }^{\mathrm{a}} \mathrm{Y}=$ massa do fruto $(\mathrm{g})$ e $\mathrm{X}=$ dias de armazenamento.

ns=não significativo; $* *=$ significativo a $1 \%$ de probabilidade.

TABELA 2 - Teste de paralelismo entre as retas estabelecidas pela relação da evolução da massa fresca, em função do tempo de armazenamento, de goiabas-'Paluma', submetidas a diferentes tratamentos e armazenadas sob condições de ambiente $\left(21,6^{\circ} \mathrm{C} ; 73,4 \% \mathrm{UR}\right)$.

\begin{tabular}{|c|c|c|c|}
\hline Tratamento & Paralelismo & Tratamento & Paralelismo \\
\hline Testemunha $\mathrm{X} \mathrm{CaCl}{ }_{2} 2 \%$ & $3,37 * *$ & $\mathrm{CaCl}_{2} 2 \%$ X $6-\mathrm{BAP} 00 \mathrm{mg} \cdot \mathrm{L}^{-1} 2$ & $7,98 * *$ \\
\hline Testemunha X 6-BAP $200 \mathrm{mg}^{-\mathrm{L}^{-1}}$ & $5,99 * *$ & $\mathrm{CaCl}_{2} 2 \% \times$ IAA $100 \mathrm{mg} . \mathrm{L}^{-1}$ & $3,51 * *$ \\
\hline Manitol $\mathrm{X} \mathrm{CaCl}_{2} 2 \%$ & $2,76^{*}$ & $\mathrm{CaCl}_{2} 2 \% \times \mathrm{IAA} 200 \mathrm{mg} \cdot \mathrm{L}^{-1}$ & $3,50 * *$ \\
\hline Manitol X 6-BAP $200 \mathrm{mg}^{-\mathrm{L}^{-1}}$ & $5,07 * *$ & GA $100 \mathrm{mg} \cdot \mathrm{L}^{-1} \times \mathrm{GA} 200 \mathrm{mg} \cdot \mathrm{L}^{-1}$ & $3,44 * *$ \\
\hline $\mathrm{CaCl}_{2} 1 \% \times \mathrm{CaCl}_{2} 2 \%$ & $2,56 *$ & GA $100 \mathrm{mg} . \mathrm{L}^{-1} \times 6-\mathrm{BAP} 200 \mathrm{mg} \cdot \mathrm{L}^{-1}$ & $7,11 * *$ \\
\hline $\mathrm{CaCl}_{2} 1 \% \times \mathrm{GA} 200{\mathrm{mg} . \mathrm{L}^{-1}}^{-1}$ & $2,39 *$ & GA $200 \mathrm{mg} . \mathrm{L}^{-1} \times 6-\mathrm{BAP} 200 \mathrm{mg} \cdot \mathrm{L}^{-1}$ & $5,12 * *$ \\
\hline $\mathrm{CaCl}_{2} 1 \% \times$ Citocinina $200 \mathrm{mg} \mathrm{L}^{-1}$ & $6,50 * *$ & 6-BAP $100 \mathrm{mg} \cdot \mathrm{L}^{-1} \times 6-$ B AP $200 \mathrm{mg} . \mathrm{L}^{-1}$ & $6,40 * *$ \\
\hline $\mathrm{CaCl}_{2} 2 \% \times \mathrm{GA} 200 \mathrm{mg} . \mathrm{L}^{-1}$ & $5,10 * *$ & $6-$ BAP $200 \mathrm{mg} \cdot \mathrm{L}^{-1}$ X AIA $100 \mathrm{mg} \cdot \mathrm{L}^{-1}$ & $5,26 * *$ \\
\hline $\mathrm{Ca} \mathrm{Cl}_{2} 2 \% \times 6-\mathrm{BAP} 200 \mathrm{mg} \cdot \mathrm{L}^{-1}$ & $3,78 * *$ & $6-$ B AP $200 \mathrm{mg} . \mathrm{L}^{-1}$ X AIA $200 \mathrm{mg} . \mathrm{L}^{-1}$ & $5,26 * *$ \\
\hline
\end{tabular}

$*=$ significativo a $5 \%$ de probabilidade e $* *=$ a $1 \%$ de probabilidade.

Obs: As outras comparações não se mostraram significativamente diferentes.

TABELA 3 - Evolução da aparência e da porcentagem de podridões em frutos de goiaba-'Paluma', submetidos a diferentes tratamentos e armazenados sob condições de ambiente $\left(21,6^{\circ} \mathrm{C} ; 73,4 \%\right.$ UR).

\begin{tabular}{|c|c|c|c|c|c|c|c|c|c|c|}
\hline \multirow[t]{2}{*}{ Tratamento } & \multicolumn{10}{|c|}{ Tempo (dia) } \\
\hline & & 1 & 2 & 3 & 4 & 5 & 6 & 7 & 8 & 9 \\
\hline \multirow[t]{2}{*}{ Testemunha } & $\mathrm{Do}^{1}$ & 0,0 & 0,0 & 0,0 & 0,0 & 0,0 & 0,0 & 0,0 & 0,0 & 0,0 \\
\hline & Ap. ${ }^{2}$ & 1,0 & 2,0 & 2,0 & 2,0 & 3,0 & 3,0 & 4,0 & 4,5 & 5,0 \\
\hline \multirow[t]{2}{*}{ Manitol } & Do & 0,0 & 0,0 & 0,0 & 0,0 & 0,0 & 0,0 & 0,0 & 0,0 & 0,0 \\
\hline & Ap. & 1,0 & 2,0 & 2,0 & 2,5 & 3,0 & 3,0 & 4,5 & 4,8 & 5,0 \\
\hline \multirow[t]{2}{*}{$\mathrm{CaCl}_{2} 1 \%$} & Do & 0,0 & 0,0 & 0,0 & 0,0 & 0,0 & 0,0 & 0,0 & 0,0 & 0,0 \\
\hline & Ap. & 1,0 & 2,0 & 2,0 & 2,0 & 3,0 & 3,0 & 3,0 & 3,5 & 4,0 \\
\hline \multirow[t]{2}{*}{$\mathrm{CaCl} 2 \%$} & Do & 0,0 & 0,0 & 0,0 & 0,0 & 0,0 & 0,0 & 0,0 & 0,0 & 0,0 \\
\hline & Ap. & 1,0 & 2,0 & 2,0 & 2,0 & 3,0 & 3,0 & 3,0 & 3,5 & 4,0 \\
\hline \multirow[t]{2}{*}{ GA $100 \mathrm{mg} \cdot \mathrm{L}^{-1}$} & Do & 0,0 & 0,0 & 0,0 & 0,0 & 0,0 & 0,0 & 0,0 & 0,0 & 0,0 \\
\hline & Ap. & 1,0 & 2,0 & 2,0 & 2,0 & 3,0 & 3,0 & 4,0 & 4,0 & 5,0 \\
\hline \multirow[t]{2}{*}{ GA $200 \mathrm{mg} \mathrm{L}^{-1}$} & Do & 0,0 & 0,0 & 0,0 & 0,0 & 0,0 & 0,0 & 0,0 & 0,0 & 6,2 \\
\hline & Ap. & 1,0 & 2,0 & 2,0 & 2,0 & 3,0 & 3,0 & 4,0 & 5,0 & 5,0 \\
\hline \multirow[t]{2}{*}{6 -B AP $100 \mathrm{mg} . \mathrm{L}^{-1}$} & Do & 0,0 & 0,0 & 0,0 & 0,0 & 0,0 & 0,0 & 0,0 & 0,0 & 0,0 \\
\hline & Ap. & 1,0 & 2,0 & 2,0 & 2,0 & 3,0 & 3,0 & 4,0 & 5,0 & 5,0 \\
\hline \multirow[t]{2}{*}{6 -B AP $200 \mathrm{mg} \cdot \mathrm{L}^{-1}$} & Do & 0,0 & 0,0 & 0,0 & 0,0 & 0,0 & 0,0 & 0,0 & 0,0 & 0,0 \\
\hline & Ap. & 1,0 & 2,0 & 2,0 & 2,0 & 3,0 & 3,0 & 4,0 & 5,0 & 5,0 \\
\hline \multirow[t]{2}{*}{ IAA $100 \mathrm{mg} \cdot \mathrm{L}^{-1}$} & Do & 0,0 & 0,0 & 0,0 & 0,0 & 0,0 & 0,0 & 0,0 & 0,0 & 0,0 \\
\hline & Ap. & 1,0 & 2,0 & 2,0 & 2,0 & 3,0 & 3,0 & 4,5 & 4,8 & 5,0 \\
\hline \multirow[t]{2}{*}{ IAA $200 \mathrm{mg} \cdot \mathrm{L}^{-1}$} & Do & 0,0 & 0,0 & 0,0 & 0,0 & 0,0 & 0,0 & 0,0 & 6,25 & 6,2 \\
\hline & Ap. & 1,0 & 2,0 & 2,0 & 2,0 & 3,0 & 3,0 & 4,5 & 5,0 & 5,0 \\
\hline
\end{tabular}

${ }^{1}$ Do=doença (\% de frutos com sintomas entre os 16 frutos avaliados).

${ }^{2} \mathrm{Ap}$.=aparência ( $1=$ fruto ótimo e $5=$ fruto totalmente murcho).

(IAA 100 e 200 mg. $\mathrm{L}^{-1}$ ) apresentaram a melhor preservação do conteúdo de ácido ascórbico.

O comportamento respiratório das goiabas não foi afetado pelos tratamentos, e a respiração, medida em $\mathrm{mgCO} \cdot \mathrm{kg}^{-1} \cdot \mathrm{h}^{-1}$, mostrou-se intensa e com aumentos sucessivos a partir de 110,83, no $1^{\circ}$ dia, atingindo 128,47 , no $7^{\circ}$ dia (Tabela 8). Não se observou um comportamento que o caracterizasse como climatérico, o que é concordante com o observado por Mattiuz et al. (2000), que também trabalhou com goiabas-'Paluma'. 
TABELA 4 - Evolução do ângulo hue ou de cor, externo, em goiabas-'Paluma', submetidas a diferentes tratamentos e armazenadas sob condições de ambiente $\left(21,6^{\circ} \mathrm{C} ; 73,4 \% \mathrm{UR}\right)$.

\begin{tabular}{|c|c|c|c|c|}
\hline \multirow[t]{2}{*}{ Tratamento } & \multicolumn{4}{|c|}{ Tempo (dia) } \\
\hline & 1 & 3 & 5 & 7 \\
\hline Testemunha & 117,0 & 98,2 & 93,6 & 91,7 \\
\hline Manitol & 117,0 & 100,9 & 96,5 & 95,6 \\
\hline $\mathrm{CaCl}_{2} 1 \%$ & 117,0 & 108,5 & 100,3 & 92,6 \\
\hline $\mathrm{CaCl}_{2} 2 \%$ & 117,0 & 107,5 & 94,6 & 97,1 \\
\hline GA $100 \mathrm{mg} \cdot \mathrm{L}^{-1}$ & 117,0 & 106,5 & 92,5 & 92,0 \\
\hline GA $200 \mathrm{mg} \cdot \mathrm{L}^{-1}$ & 117,0 & 103,4 & 95,5 & 92,9 \\
\hline 6-BAP 100 mg.L ${ }^{-1}$ & 117,0 & 106,5 & 100,1 & 95,3 \\
\hline 6-BAP $200 \mathrm{mg} \cdot \mathrm{L}^{-1}$ & 117,0 & 107,5 & 97,7 & 96,9 \\
\hline IAA $100 \mathrm{mg} . \mathrm{L}^{-1}$ & 117,0 & 108,0 & 96,0 & 92,3 \\
\hline IAA $200 \mathrm{mg} \cdot \mathrm{L}^{-1}$ & 117,0 & 105,0 & 96,6 & 91,3 \\
\hline Média & $117,0 \mathrm{a}$ & $105,2 b$ & $96,3 c$ & $93,3 d$ \\
\hline
\end{tabular}

$\mathrm{dms}=2,22 ; \mathrm{CV}=3,82 \%$.

Médias seguidas de pelo menos uma letra comum não diferem entre si, ao nível de $5 \%$ de probabilidade, pelo teste de Tukey.

TABELA 5 - Evolução da firmeza, em Newtons, em frutos de goiaba-'Paluma', submetidos a diferentes tratamentos e armazenados sob condições de ambiente $\left(21,6^{\circ} \mathrm{C} ; 73,4 \% \mathrm{UR}\right)$.

\begin{tabular}{lcccc}
\hline Tratamento & \multicolumn{3}{c}{ Tempo (dia) } & \\
\cline { 2 - 5 } & 1 & 3 & 5 & 7 \\
Testemunha & 97,3 & 29,2 & 28,3 & 21,0 \\
Manitol & 97,3 & 41,0 & 22,4 & 18,3 \\
$\mathrm{CaCl}_{2}$ 1\% & 97,3 & 58,2 & 28,9 & 20,5 \\
$\mathrm{CaCl}_{2} 2 \%$ & 97,3 & 29,6 & 21,9 & 24,0 \\
GA 100 mg.L-1 & 97,3 & 38,6 & 18,5 & 23,3 \\
GA 200 mg.L-1 & 97,3 & 36,7 & 33,1 & 25,0 \\
6-BAP 100 mg.L-1 & 97,3 & 32,5 & 32,9 & 19,3 \\
6-BAP 200 mg.L-1 & 91,6 & 32,9 & 16,5 \\
IAA 100 mg.L-1 & 97,3 & 36,4 & 37,1 & 16,8 \\
IAA 200 mg.L-1 & 97,3 & 32,6 & $24,4 \mathrm{c}$ & 21,0 \\
Média & 97,3 & $37,6 \mathrm{~b}$ & & $19,6 \mathrm{~d}$ \\
\hline
\end{tabular}

$\mathrm{dms}=7,55 ; \mathrm{CV}=19,58 \%$.

Médias seguidas de pelo menos uma letra comum não diferem entre si, ao nível de $5 \%$ de probabilidade, pelo teste de Tukey.

TABELA 6 - Evolução no conteúdo de pectina total e solúvel em goiabas-'Paluma', submetidas a diferentes tratamentos e armazenadas sob condições de ambiente $\left(21,6^{\circ} \mathrm{C} ; 73,4 \%\right.$ UR).

\begin{tabular}{|c|c|c|c|c|c|c|}
\hline \multirow{3}{*}{ Tratamento } & \multicolumn{6}{|c|}{ Tempo (dia) } \\
\hline & \multicolumn{2}{|c|}{ Pectina Total } & \multicolumn{4}{|c|}{ Pectina Solúvel } \\
\hline & 1 & 7 & 1 & 3 & 5 & 7 \\
\hline Testemunha & 0,89 & 0,69 & $0,12 \mathrm{a}$ & $0,32 \mathrm{ab}$ & $0,47 \mathrm{ab}$ & $0,50 \mathrm{~b}$ \\
\hline Manitol & 0,89 & 0,58 & $0,12 \mathrm{a}$ & $0,30 \mathrm{ab}$ & $0,43 \mathrm{~b}$ & $0,41 \mathrm{~cd}$ \\
\hline $\mathrm{CaCl}_{2} 1 \%$ & 0,89 & 0,66 & $0,12 \mathrm{a}$ & $0,29 b c$ & $0,35 \mathrm{~d}$ & $0,43 \mathrm{~cd}$ \\
\hline $\mathrm{CaCl}_{2} 2 \%$ & 0,89 & 0,66 & $0,12 \mathrm{a}$ & $0,28 \mathrm{bc}$ & $0,44 b$ & $0,44 \mathrm{c}$ \\
\hline GA $100 \mathrm{mg} \cdot \mathrm{L}^{-1}$ & 0,89 & 0,70 & $0,12 \mathrm{a}$ & $0,26 \mathrm{~cd}$ & $0,40 \mathrm{~cd}$ & $0,42 \mathrm{c}$ \\
\hline GA $200 \mathrm{mg} \cdot \mathrm{L}^{-1}$ & 0,89 & 0,75 & $0,12 \mathrm{a}$ & $0,23 \mathrm{~d}$ & $0,31 \mathrm{e}$ & $0,57 \mathrm{a}$ \\
\hline 6-B AP $100 \mathrm{mg} \cdot \mathrm{L}^{-1}$ & 0,89 & 0,74 & $0,12 \mathrm{a}$ & $0,34 \mathrm{a}$ & $0,42 \mathrm{bc}$ & $0,40 \mathrm{c}$ \\
\hline $6-$ B AP $200 \mathrm{mg}^{-\mathrm{L}^{-1}}$ & 0,89 & 0,75 & $0,12 \mathrm{a}$ & $0,33 \mathrm{a}$ & $0,49 \mathrm{a}$ & $0,42 \mathrm{~cd}$ \\
\hline IA A $100 \mathrm{mg} \cdot \mathrm{L}^{-1}$ & 0,89 & 0,63 & $0,12 \mathrm{a}$ & $0,26 \mathrm{~cd}$ & $0,39 \mathrm{~cd}$ & $0,39 d$ \\
\hline IA A $200 \mathrm{mg} \cdot \mathrm{L}^{-1}$ & 0,89 & 0,53 & $0,12 \mathrm{a}$ & $0,25 \mathrm{~cd}$ & $0,43 \mathrm{bc}$ & $0,41 \mathrm{~cd}$ \\
\hline
\end{tabular}

Resultados expressos em grama de ácido urônico.100g de polpa ${ }^{-1}$.

$\mathrm{dms}=0,0402 ; \mathrm{CV}=3,81 \%$.

Médias seguidas de pelo menos uma letra comum, nas colunas, não diferem entre si, ao nível de 5\% de probabilidade, pelo teste de Tukey. 
TABELA 7 - Evolução do conteúdo de ácido ascórbico, expresso em mg de ácido ascórbico.100g de polpa-1 , em goiabas-'Paluma', submetidas a diferentes tratamentos e armazenadas sob condições de ambiente $\left(21,6^{\circ} \mathrm{C} ; 73,4 \% \mathrm{UR}\right)$.

\begin{tabular}{|c|c|c|c|c|}
\hline \multirow[t]{2}{*}{ Tratamento } & \multicolumn{4}{|c|}{ Tempo (dia) } \\
\hline & 1 & 3 & 5 & 7 \\
\hline Testemunha & $78,5 \mathrm{a}$ & $53,4 \mathrm{~g}$ & $48,0 \mathrm{f}$ & $42,2 \mathrm{e}$ \\
\hline Manitol & $78,5 \mathrm{a}$ & $76,8 \mathrm{a}$ & $65,8 \mathrm{bc}$ & $56,9 \mathrm{bc}$ \\
\hline $\mathrm{CaCl}_{2} 1 \%$ & $78,5 \mathrm{a}$ & $64,8 \mathrm{c}$ & $62,9 c$ & $58,4 b$ \\
\hline $\mathrm{CaCl}_{2} 2 \%$ & $78,5 \mathrm{a}$ & $61,6 \mathrm{cde}$ & $58,4 \mathrm{de}$ & $55,8 \mathrm{bcd}$ \\
\hline GA $100 \mathrm{mg} . \mathrm{L}^{-1}$ & $78,5 \mathrm{a}$ & $62,7 \mathrm{~cd}$ & $58,1 \mathrm{de}$ & $56,8 \mathrm{bc}$ \\
\hline GA $200 \mathrm{mg} \cdot \mathrm{L}^{-1}$ & $78,5 \mathrm{a}$ & $58,2 \mathrm{ef}$ & $57,5 \mathrm{de}$ & $54,1 \mathrm{~cd}$ \\
\hline 6-B AP $100 \mathrm{mg}^{-\mathrm{L}^{-1}}$ & $78,5 \mathrm{a}$ & $60,1 \mathrm{def}$ & $59,0 \mathrm{~d}$ & $57,1 \mathrm{bc}$ \\
\hline $6-\mathrm{BAP} 200 \mathrm{mg} \cdot \mathrm{L}^{-1}$ & $78,5 \mathrm{a}$ & $58,1 \mathrm{f}$ & $55,4 \mathrm{e}$ & $53,0 \mathrm{~d}$ \\
\hline IAA $100 \mathrm{mg} \cdot \mathrm{L}^{-1}$ & $78,5 \mathrm{a}$ & $76,9 a$ & $67,4 b$ & $66,9 a$ \\
\hline IAA $200 \mathrm{mg} . \mathrm{L}^{-1}$ & $78,5 \mathrm{a}$ & $71,6 b$ & $71,9 \mathrm{a}$ & $68,8 \mathrm{a}$ \\
\hline
\end{tabular}

$\mathrm{dms}=3,49 ; \mathrm{CV}=1,6 \%$.

Médias seguidas de pelo menos uma letra comum, nas colunas, não diferem entre si, ao nível de 5\% de probabilidade, pelo teste de Tukey.

TABELA 8 - Produção de $\mathrm{CO}_{2}\left(\mathrm{mgCO}_{2} \cdot \mathrm{kg}^{-1} \cdot \mathrm{h}^{-1}\right)$ por goiabas-'Paluma', submetidas a diferentes tratamentos e armazenadas sob condições de ambiente $\left(21,6^{\circ} \mathrm{C} ; 73,4 \% \mathrm{UR}\right)$.

\begin{tabular}{|c|c|c|c|c|c|c|c|}
\hline \multirow[t]{2}{*}{ Tratamento } & \multicolumn{7}{|c|}{ Tempo (dia) } \\
\hline & 1 & 2 & 3 & 4 & 5 & 6 & 7 \\
\hline Testemunha & 110,83 & 95,71 & 140,09 & 129,26 & 140,11 & 150,55 & 132,51 \\
\hline Manitol & 110,83 & 123,93 & 115,30 & 109,00 & 119,31 & 131,46 & 131,47 \\
\hline $\mathrm{CaCl}_{2} 1 \%$ & 110,83 & 78,02 & 114,27 & 116,30 & 127,40 & 145,79 & 121,21 \\
\hline $\mathrm{CaCl}_{2} 2 \%$ & 110,83 & 111,07 & 135,62 & 136,02 & 142,12 & 143,82 & 137,78 \\
\hline GA $100 \mathrm{mg} \cdot \mathrm{L}^{-1}$ & 110,83 & 112,89 & 107,60 & 124,62 & 139,55 & 132,11 & 121,21 \\
\hline GA $200 \mathrm{mg} . \mathrm{L}^{-1}$ & 110,83 & 93,70 & 103,85 & 92,26 & 109,51 & 124,23 & 103,28 \\
\hline 6-BAP $100 \mathrm{mg} \cdot \mathrm{L}^{-1}$ & 110,83 & 89,56 & 112,96 & 159,50 & 166,03 & 199,32 & 136,69 \\
\hline 6-BAP $200 \mathrm{mg} \cdot \mathrm{L}^{-1}$ & 110,83 & 108,92 & 91,51 & 121,69 & 146,17 & 138,40 & 133,65 \\
\hline IAA $100 \mathrm{mg} \cdot \mathrm{L}^{-1}$ & 110,83 & 120,21 & 120,12 & 118,46 & 148,44 & 149,43 & 135,78 \\
\hline IAA $200 \mathrm{mg} . \mathrm{L}^{-1}$ & 110,83 & 115,11 & 112,06 & 133,46 & 140,42 & 134,37 & 131,14 \\
\hline
\end{tabular}

\section{CONCLUSÕES}

Os tratamentos com cloreto de cálcio a $1 \%$ propiciaram vida útil de 7 dias às goiabas- 'Paluma', com manutenção da aparência e menor perda de massa fresca. Os tratamentos utilizados não influenciaram na evolução da coloração e da firmeza.

\section{REFERÊNCIAS BIBLIOGRÁFICAS}

ASSOCIATION OF OFFICIAL ANALYTICAL CHEMISTS INTERNATIONAL. Official methods of analysis of the AOAC international. 16 ed. Washington, 1997.v.2, p.6, 11, 16.

BARNETT, H. L.; HUNTER, B. B. Ilustrated genera of imperfect fungi. 3. ed. Minneapolis: Beugess Pub., 1972. 241p.

BLUMENKRANTZ, N.; ASBOE-HANSEN, G. New method for quantitative determination of uronic acids. Analitical Chemistry, New York, v.5, p.484-489, 1973.

BRADY, C. J. Fruit ripening. Annual Review of Plant Physiology, California, n.38, p.155-177. 1987.

CHITARRA, M. I. F; CHITARRA, A. B.Pós-colheita de frutos e hortaliças: fisiologia e manuseio. Lavras-MG: FAEP, 1990. 230p.

DURIGAN, J. F. Colheita, conservação e embalagens. In: SIMPÓSIO BRASILEIRO SOBRE A CULTURA DA GOIABEI-
RA, 1., 1997, Jaboticabal. Anais...Jaboticabal: FUNEP, 1997. p.152154.

FRENKEL, C.; KLEIN, I.; DILLEY, D. R. Methods for the study of ripening and protein synthesis in intact pome fruits. Phytochemistry, Oxford, v.8, p.945-955, 1969.

GOMES, F. P. Curso de estatística experimental. 7. ed. Piracicaba: Nobel, 1977. 430p.

JACOMINO, A. P.; SIGRIST, J. M. M.; SARANTÓPOULOS, C. I. G. L.; MINAMI, K.; KLUGE, R. A. Embalagens para conservação refrigerada de goiabas. Revista Brasileira de Fruticultura, Jaboticabal, v. 23, n.1 , p.50-54, 2001.

LIMA, M. A.; DURIGAN, J. F. Conservação de goiabas 'Pedro Sato' associando-se refrigeração com diferentes embalagens plásticas. Revista Brasileira de Fruticultura, Jaboticabal, v. 22, n.2, p.232-235, 2000.

LIMA, M. A.; Conservação pós-colheita e caracterização tecnológica dos frutos de diferentes genótipos de goiaba (Psidium guajava L.) produzidos em Jaboticabal-SP, 1999. 101p. Dissertação (Mestrado) - Faculdade de Ciências Agrárias e Veterinárias, Universidade Estadual Paulista, Jaboticabal, 1999.

LUDFORD, P. L. Postharvest hormone changes in vegetables and fruits. In: DAVIES, P. J. (Ed.) Plant hormones. The Netherlands: Kluwer Ac. Pub., 1995. p. 725-750. 
MATTIUZ,B-H; DURIGAN, J. F.; TEIXEIRA, G. H. de A. Efecto de la injuria mecánica de impacto en la calidad poscosecha de guayaba 'Paluma'. Revista Iberoamericana de Tecnologia Postcosecha, Sonora, v.2, n. 2, p.115-120, 2000.

McCREADY, R. M.; McCOMB, E. A. Extraction and determination of total pectic materials. Analytical Chemistry, Washington, v.24, n.12, p.1586-1588, 1952.

NETER, J.; WASSERMAN, W.; WHITMORE, G. A. Applied linear statistical models. Massachussetts: Allyn and Bacon, IAC, 1978.745p.

PATHMANABAN, G.; NAGARAJAN, M.; MANIAN, K.; ANNAMALAINATHAN, K. Effect of fused calcium salts on post harvest preservation in fruits. Madras Agricultural Journal, Coimbatore, v.82, n.1, p.47-50, 1995.
PEREIRA, F. M. Cultura da goiabeira. Jaboticabal:FUNEP, 1995. $47 \mathrm{p}$.

SIDDIQUI, S.; BANGERTH, F. The effect of calcium infiltration on structural changes in cell walls of stored apples. Journal of Horticultural Science, Ashford, v.5, n.71, p.703-708, 1996.

TEAOTIA, S. S.; TRIPATH, C. S.; SINGH, R. N. Effect of growth substances on ripening and quality of guava (Psidium guajava L.). Journal Food Science Technology, Chicago, n.9, p.38, 1972.

VASQUEZ-OCHOA, R. I.; COLINAS-LEON, M. T. Changes in guavas of three maturity stages in response to temperature and relative humidity. HortScience, México, v.25, n.1, 1990. p.86-87.

VENDRELL, M. Dual effect of 2,4-D on ethylene production and ripening of tomato fruit tissue. Physiologia Plantarum, Copenhagem, n.64, p.559-563, 1985. 